Supporting Information for:

\title{
Presentation and Recognition of Biotin on Nanofibers Formed by Branched Peptide Amphiphiles
}

\author{
Mustafa O. Guler ${ }^{\dagger}$, Stephen Soukasene ${ }^{\ddagger}$, James F. Hulvat ${ }^{\ddagger}$ and Samuel I. Stupp ${ }^{\star}$, ,, , \\ 'Department of Chemistry, ${ }^{*}$ Department of Materials Science and \\ Engineering, ${ }^{s}$ Feinberg School of Medicine \\ Northwestern University \\ 2020 Campus Drive \\ Evanston, IL 60208
}

\section{Experimental Section.}

\author{
Abbreviations. \\ Fmoc......9-Fluorenylmethoxycarbonyl \\ Boc........ter. Butoxycarbonyl \\ Mtt.........4-Methyltrityl \\ HBTU......2-(1H-Benzotriazol-1-yl)-1,1,3,3-tetramethyluronium hexafluorophosphate \\ FITC........Fluorescein Isothiocyanate \\ DIEA.......N,N-Diisopropylethylamine
}

\section{Materials.}

Fmoc, Boc and 4-Methyltrityl (Mtt) protected amino acids, MBHA Rink Amide resin, and HBTU were purchased from NovaBiochem. Bodipy-NeutrAvidin was purchased from Molecular Probes. The other chemicals were purchased from Fisher or Aldrich and used as provided.

\section{Synthesis of Peptides.}

Peptides were constructed on MBHA Rink Amide resin. Amino acid couplings were done with 4 equivalents of Fmoc protected amino acid, 3.95 equivalents HBTU and 6 equivalents of DIEA for $4 \mathrm{~h}$. Fmoc deprotections were performed with $30 \%$ Piperidine / DMF solution for 10 min. Mtt removal was done with 4\% TFA / Dichloromethane solution in the presence of TIS for 5 min. Cleavage of the peptides from the resin was carried out with a mixture of TFA:TIS: $\mathrm{H}_{2} \mathrm{O}$ in ratio of 95:2.5:2.5 for $3 \mathrm{~h}$. Excess TFA was removed by rotary evaporation. The remaining viscous peptide solution was triturated with cold ether and the resulting white product was dried under vacuum. PAs were characterized by Matrix Assisted laser desorption ionization-time of flight mass spectrometry (MALDI-TOF MS) and Electrospray Ionization Mass Spectrometry (ESIMS).

\section{Peptide Amphiphile Design.}

The branched PAs were prepared by Fmoc solid phase peptide synthesis (SPPS), and branching of the peptide head group was achieved using orthogonal protecting group 
chemistry for the amines at the $\alpha$ and $\varepsilon$ positions of the lysine residue. First, FmocLys(Mtt)-OH, (Mtt: 4-methyl trityl) was coupled to MBHA Rink Amide resin, followed by cleavage of the Mtt protecting group on the $\varepsilon$ amine to couple palmitic acid without affecting Fmoc protection. ${ }^{14}$ This was followed by Fmoc removal on the $\alpha$ amine to grow the peptide segment of the PA. The branching point in PAs was introduced at a lysine dendron using Fmoc-Lys(Mtt)-OH. To grow the first arm of PAs, Fmoc on the $\alpha$ amine was removed before Mtt. Boc-Lys(Boc)-OH was coupled at the end of the first branch. Both $\alpha$ and $\varepsilon$ amine positions of the lysine were blocked with Boc protection as it is more stable under the cleavage conditions employed for the removal of Fmoc and Mtt. Later, Mtt was removed and the other branch was grown using Boc-Lys(Fmoc)-OH. As mentioned before the bioactive peptide sequence was built on the branch (using the $\varepsilon$ amine) in order to probe enhanced presentation and recognition by the avidin receptor. Two identical arms were formed with Fmoc-Lys(Fmoc)-OH at the branching point. Both arms were grown at the same time. Biotinylated branched PAs were synthesized by coupling biotin to the end of the peptide sequence via Fmoc SPPS.

\section{Transmission Electron Microscopy (TEM) and Atomic Force Microscopy (AFM) samples.}

The TEM samples were prepared from 1 wt \% gels of the PAs on a holey carbon coated TEM grid. Negative staining was done with $2 \mathrm{wt} \%$ phosphotungstic acid in water.

The AFM samples were prepared by casting $10 \mathrm{~mL}$ of a $0.10 \mathrm{wt} \%$ aqueous solution of the molecule onto a (111) silicon surface (Silicon Quest International, Inc., Santa Clara, CA) that had been cleaned by $15 \mathrm{~min}$. ultrasonication in water (Millipore filtered, resistivity $>18.2 \mathrm{MW} \times \mathrm{cm}$ ) and isopropanol (HPLC purity, Fisher Scientific). After evaporation of water, tapping mode atomic force microscopy (AFM) was performed using a Digital Instruments MultiMode microscope (TESPA7 tapping tips, Vecco Nanoprobes).

\section{Circular Dichroism (CD) spectroscopy.}

The CD samples were prepared at $\mathrm{pH} 7.4$ at the concentrations of $3.33 \times 10^{-6} \mathrm{M}$ in a $1 \mathrm{~mm}$ quartz cell.

\section{Fluorescence spectroscopy.}

The commercially available BODIPY-NeutrAvidin and FITC-avidin was used to study fluorescence emission change upon binding of biotinylated PAs. PA samples were first prepared at $\mathrm{pH} 7.4$ at $2 \times 10^{-7} \mathrm{M}$ and $5 \times 10^{-8} \mathrm{M}$ Bodipy-NeutrAvidin was added. For FITC-avidin samples, PA samples were first prepared at $\mathrm{pH} 7.4$ at $2 \times 10^{-8} \mathrm{M}$ and $5 \times 10^{-9} \mathrm{M}$ FITC-avidin was added. 


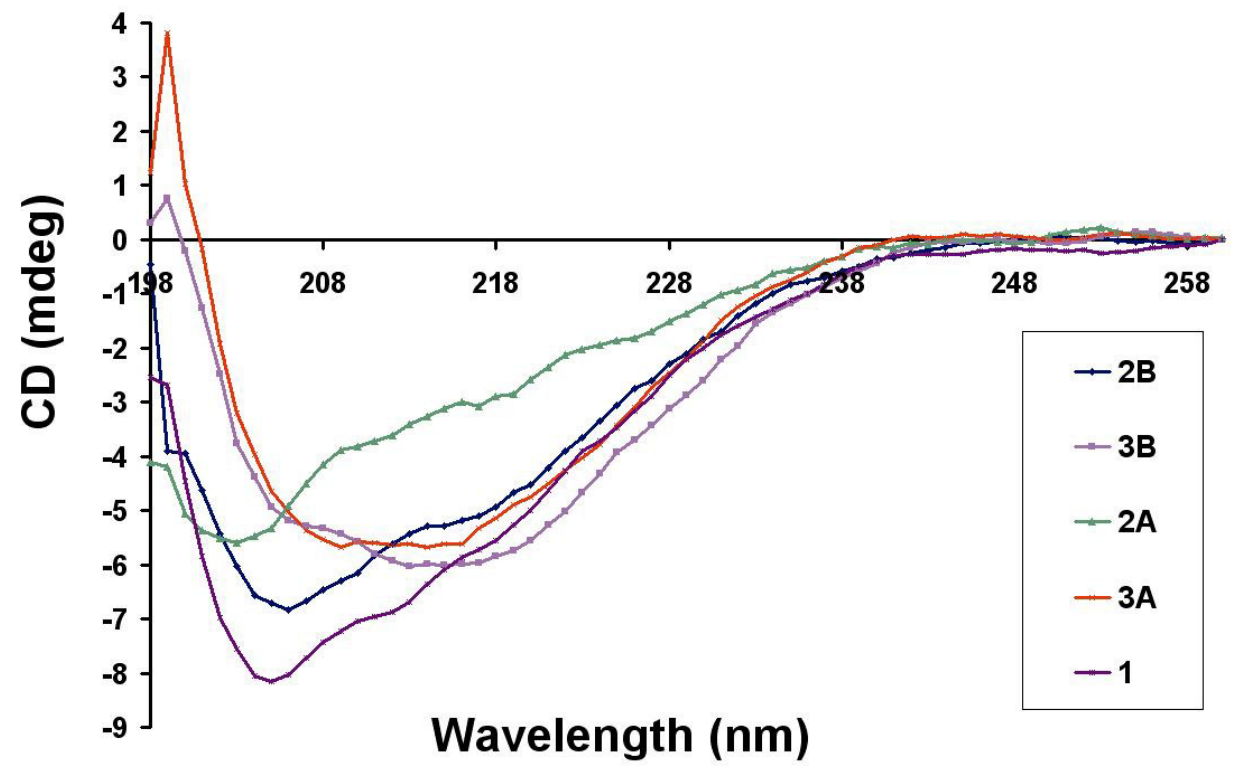

Figure S1. Circular Dichorism Data of the PAs at PH 7.4. 


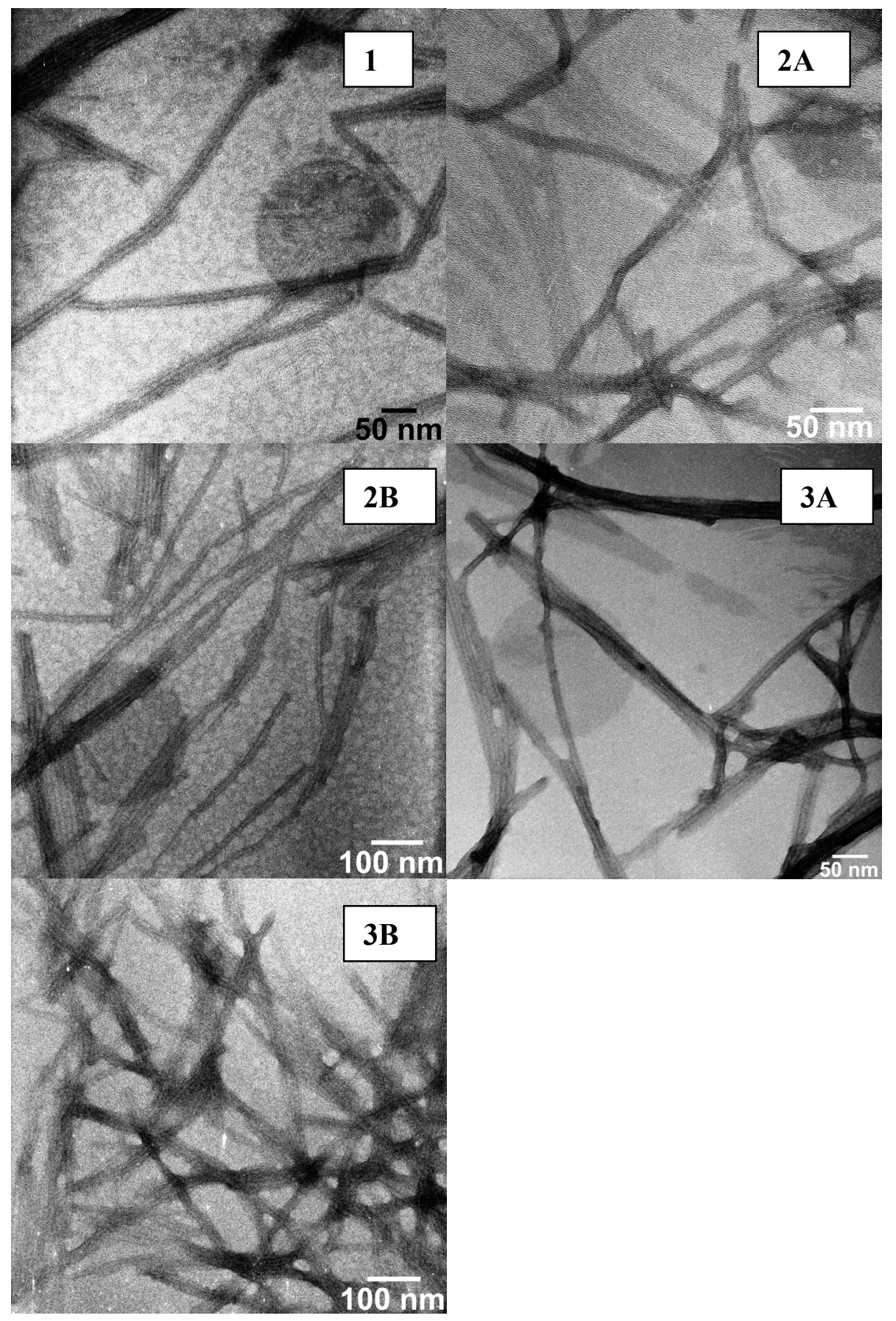

Figure S2. Negatively stained TEM micrographs of 1-3. 


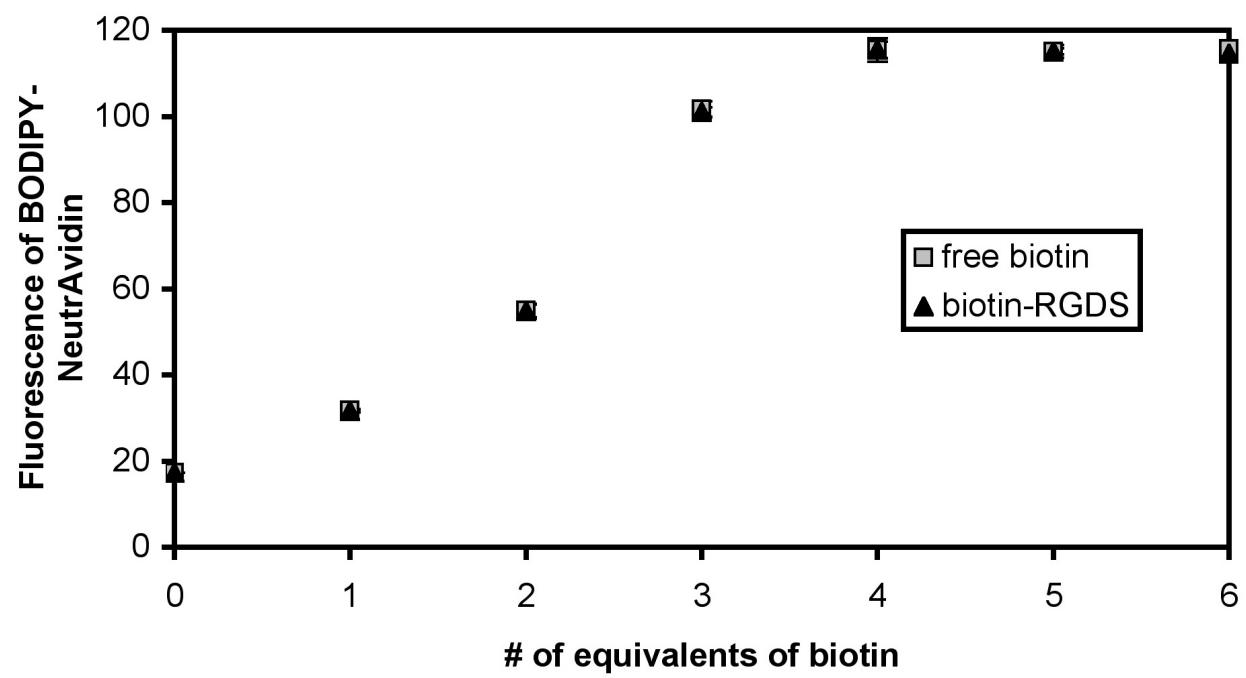

Figure S3. Increase in fluorescence emission at $514 \mathrm{~nm}$ upon binding of biotin and biotin-RGDS to BODIPY-NeutrAvidin. Error bars are a $99 \%$ confidence interval on the mean for each set of samples.

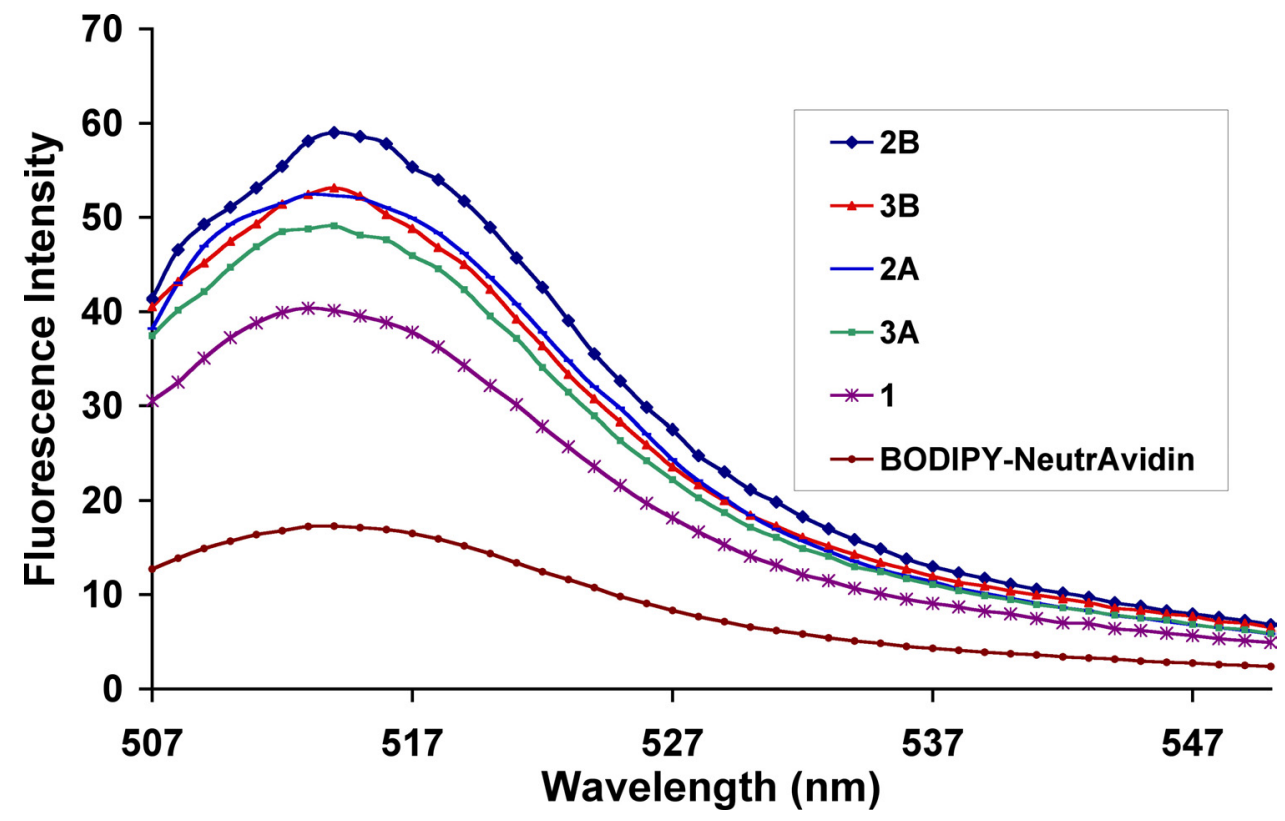

Figure S4. Fluorescence emission spectra of BODIPY-NeutrAvidin and BODIPYNeutrAvidin with PAs excited at $505 \mathrm{~nm}$ in $\mathrm{pH}$ 7.4. 


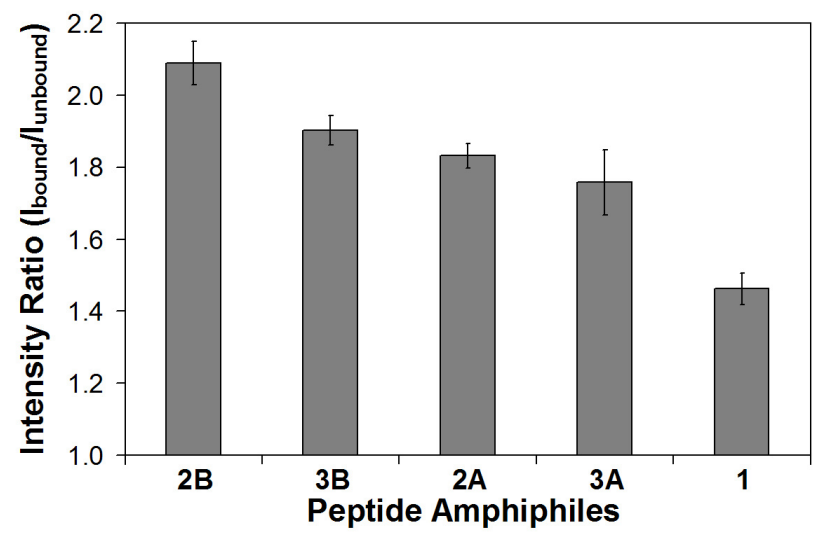

Figure S5. Increase in fluorescence emission at $520 \mathrm{~nm}$ upon binding of FITC-avidin to the biotinylated branched and linear peptide amphiphiles, relative to unbound FITCavidin at the same concentration. Error bars are a 95\% confidence interval on the mean for each set of samples.

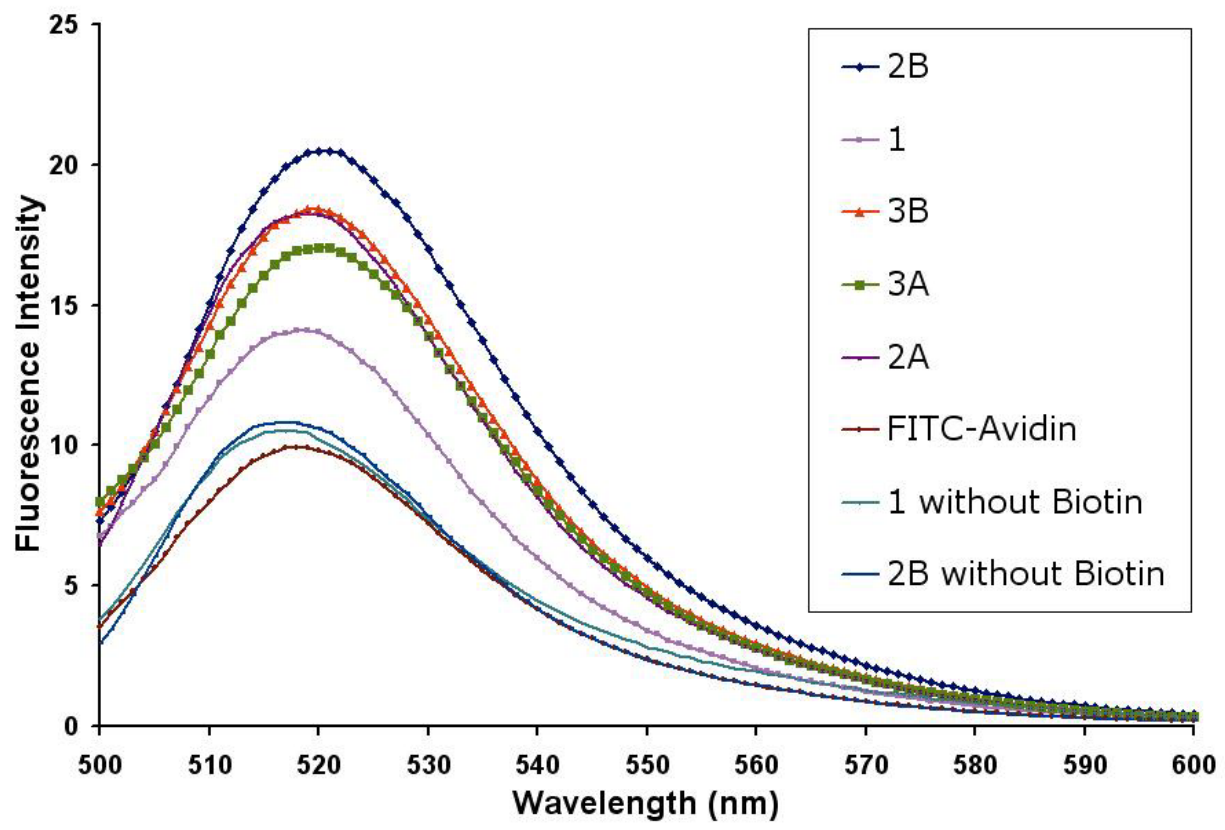

Figure S6. Fluorescence emission spectra of FITC-Avidin and FITC-Avidin with PAs excited at $490 \mathrm{~nm}$ in $\mathrm{pH} 7.4$. 


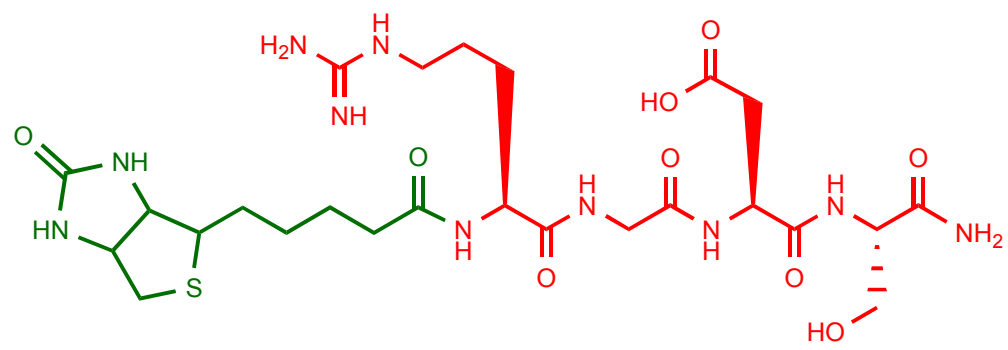

Biotin-RGDS

Figure S7. Biotin-RGDS peptide sequence as a peptide control. 